\section{Commentary: Understanding principal strain need not be stressful}

\author{
James A. DiNardo, MD, FAAP
}

Castelvecchio and colleagues ${ }^{1}$ make use of sophisticated 3dimensional ultrasound strain analysis to study left ventricular function recovery in patients with ischemic heart failure undergoing surgical remodeling. This study is a remarkable example of the use of 3-dimensional echocardiography coupled with speckle-tracking technology to measure myocardial strain. It would likely be helpful for most readers to review the concepts of stress and strain. Stress is defined as the force applied to an object that causes a change in the object. Strain is defined as the change in the shape (in this case the length of a segment of myocardium) of an object when stress is applied. Stress is measurable and has the unit of force/area, whereas strain is dimensionless. In a contracting myocardium, strain is multidimensional; radial thickening/ thinning of the left ventricle wall in systole and diastole, longitudinal (the systolic shortening and diastolic expansion of the left ventricle along its long axis), and circumferential (torsion of the left ventricle in the short axis).

In structural engineering, principal strain analysis (PSA) is commonly used to describe this type of multidimensional deformation. The authors apply this analysis to overcome the limitations of analysis of the individual strain parameters. The foremost limitation of the multidimensional approach is the individual components of strain do not correspond to any anatomic fiber orientation. PSA allows these 3 components of strain plus their 6 shear strains to be resolved into a primary strain and 1 very small secondary strain. ${ }^{2,3}$ The beauty of primary strain is that its orientation recapitulates the helical orientation of the myocardial fibers and thus provides direct measurement of anatomic fiber shortening. ${ }^{4}$ PSA

\footnotetext{
From the Division of Cardiac Anesthesia, Harvard Medical School, and Department of Anesthesiology, Boston Children's Hospital, Boston, Mass.

Disclosures: The author reported no conflicts of interest.

The Journal policy requires editors and reviewers to disclose conflicts of interest and to decline handling or reviewing manuscripts for which they may have a conflict of interest. The editors and reviewers of this article have no conflicts of interest

Received for publication Feb 14, 2021; revisions received Feb 14, 2021; accepted for publication Feb 17, 2021; available ahead of print Feb 22, 2021

Address for reprints: James A. DiNardo, MD, FAAP, Department of Anesthesiology, Boston Children's Hospital, 300 Longwood Ave, Boston, MA 02115 (E-mail: james.dinardo@childrens.harvard.edu).

J Thorac Cardiovasc Surg 2023;165:1430-1

0022-5223/\$36.00

Copyright (c) 2021 by The American Association for Thoracic Surgery

https://doi.org/10.1016/j.jtcvs.2021.02.062
}

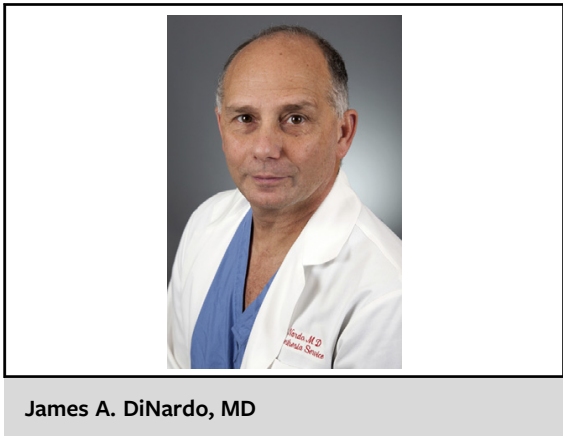

CENTRAL MESSAGE

Principal strain analysis using 3-dimensional echocardiography with speckle tracking allows assessment of myocardial strain oriented by the anatomic arrangement of myofibrils.

quantifies the mechanistic link between maximal fiber shortening and myocardial fiber arrangement.

The authors utilized PSA to measure mechanical dispersion, which is the standard deviation of multiple measurements of the time to peak shortening in a region of myocardium. This allowed them to quantify dispersion of volumetric change in terms of fiber shortening. It is telling that mechanical dispersion as assessed by 2 -dimensional longitudinal strain analysis is associated with an enhanced arrhythmogenic risk due to inhomogeneous electrical conduction and repolarization. ${ }^{5}$ Obviously, PSA allows a more comprehensive assessment of the cardiac electromechanical connection.

The number of patients studied here was small and PSA technology was used to study a very specific patient cohort. The improvement of left ventricle performance primarily in the remote basal regions of myocardium is a fascinating finding. Unfortunately, this study provides us with little insight into the mechanism of that improvement nor is the observation generalizable to other causes of reduced left ventricle function. Finally, it remains to be seen how use of PSA can be used to improve operative techniques with the intent to ultimately improve patient outcomes and quality of life.

\section{References}

1. Castelvecchio S, Frigelli M, Sturla F, Milani V, Pappalardo OA, Citarella M, et al. Elucidating the mechanisms underlying left ventricular function recovery in ischemic heart failure patients undergoing surgical remodeling: a 3D ultrasound analysis. J Thorac Cardiovasc Surg. 2023;165:1418-29.e4. 
2. Pedrizzetti G, Sengupta S, Caracciolo G, Park CS, Amaki M, Goliasch G, et al. Three-dimensional principal strain analysis for characterizing subclinical changes in left ventricular function. J Am Soc Echocardiog. 2014;27:1041-10450.e1.

3. Sato T, Calderon RJ, Klas B, Pedrizzetti G, Banerjee A. Simultaneous volumetric and functional assessment of the right ventricle in hypoplastic left heart syndrome after Fontan palliation, utilizing 3-dimensional speckle-tracking echocardiography. Circ J. 2020;84:235-44.
4. Mangual JO, Luca AD, Toncelli L, Domenichini F, Galanti G, Pedrizzetti G Three-dimensional reconstruction of the functional strain-line pattern in the left ventricle from 3-dimensional echocardiography. Circ Cardiovasc Imaging. 2012;5:808-9.

5. Haugaa KH, Grenne BL, Eek CH, Ersøll M, Valeur N, Svendsen JH, et al. Strain echocardiography improves risk prediction of ventricular arrhythmias after myocardial infarction. JACC Cardiovasc Imaging. 2013;6:841-50.
See Article page 1418.

\section{Commentary: Elucidating the mechanisms underlying left ventricular function recovery in patients with ischemic heart failure undergoing surgical remodeling: Physiology versus Empiricism}

Giuseppe Ambrosio, $\mathrm{MD}, \mathrm{PhD},{ }^{\mathrm{a}}$ Roberto Mattioli, MD, ${ }^{b}$ and Erberto Carluccio, $\mathrm{MD}^{\mathrm{a}}$

Myocardial infarction (MI) is a major etiologic factor for heart failure (HF). In addition to muscle loss, MI alters left ventricular (LV) size and shape ("remodeling") through infarct expansion, scar formation, and hypertrophy of LV remote areas. ${ }^{1,2} \mathrm{LV}$ dilatation also increases wall stress, which further decreases pump efficiency and blunts the benefits of revascularization.

Surgical "reshaping" to revert postinfarction LV dilatation and remodeling has long been advocated. ${ }^{4}$ However, the ultimate benefits have been questioned, with surgeons wondering whether its aim is just achieving LV volume reduction, or restoring a more conical shape. ${ }^{5}$ Also, the

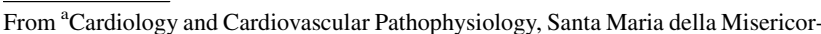
dia Hospital, University of Perugia, Perugia; and ${ }^{\mathrm{b}}$ IRCCS Multimedica, Milan, Italy.

Disclosures: The authors reported no conflicts of interest.

The Journal policy requires editors and reviewers to disclose conflicts of interest and to decline handling or reviewing manuscripts for which they may have a conflict of interest. The editors and reviewers of this article have no conflicts of interest.

Received for publication March 2, 2021; revisions received March 2, 2021; accepted for publication March 2, 2021; available ahead of print March 5, 2021.

Address for reprints: Giuseppe Ambrosio, MD, PhD, Cardiology and Cardiovascular Pathophysiology, Santa Maria della Misericordia Hospital, University of Perugia, Piazzale Menghini, 06132 Perugia, Italy (E-mail: giuseppe.ambrosio@ ospedale. perugia.it).

J Thorac Cardiovasc Surg 2023;165:1431-2

$0022-5223 / \$ 36.00$

Copyright (C) 2021 by The American Association for Thoracic Surgery

https://doi.org/10.1016/j.jtcvs.2021.03.006
}

Check for updates

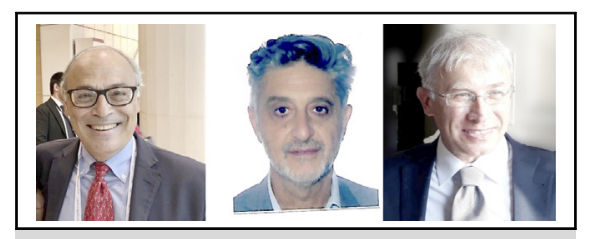

Giuseppe Ambrosio, MD, PhD, Roberto Mattioli, $\mathrm{MD}$, and Erberto Carluccio, MD

\begin{abstract}
CENTRAL MESSAGE
Why just reshaping! The importance of remote myocardium in enhancing LV function after ventricular surgical reconstruction is highlighted by 3D speckle tracking echocardiography.
\end{abstract}

STICH (Surgical Treatment for Ischemic Heart Failure) trial failed to demonstrate a clear benefit of adding LV surgical reconstruction to coronary artery bypass grafting in patients after an anterior $\mathrm{MI}^{6}$; still, that trial raised one important issue: would the benefits of surgical reduction of LV volume be offset by impaired diastolic distensibility/increased LV stiffness?

Choosing the optimal parameters to gauge improvement in LV function postoperatively is crucial when investigating the benefits of LV reshaping. Empirically, this has been pursued through measurements of LV ejection fraction (EF). That approach might prove fallacious, as EF is influenced by LV load conditions and other factors; more importantly, in this specific setting EF can be misleading. In fact, even if stroke volume remained unchanged after surgery (ie, no improvement of function), nonetheless expressing it relative to diastolic LV volume (which decreases postsurgery) would artifactually translate into greater EF; that would be just a "math improvement," not a physiologically relevant benefit. 